\section{Discoloration difference between acrylic artificial teeth exposed to filter- and electronic-cigarette smoke in the Laboratory Faculty of Dentistry, Muslim University of Indonesia in 2017}

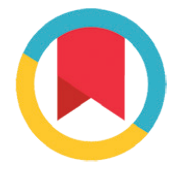

CrossMark

\author{
Chusnul Chotimah, ${ }^{*}$ Mohammad A. Ramadan
}

\begin{abstract}
Objective: To determine of artificial teeth color discoloration different between exposure of cigarette's and electric cigarette's smoke in the Laboratorium FKG UMI 2017. The simulator of smoking, artificial teeth, cigarette's, CIE Lab.

Material and Methods: Acrylic is most commonly used material for articial teeth. Acrylic is easily stained by food, beverage, chemical, and smoking habit. Cigarette and electric cigarette are commonly consumed by most people. Cigarette contains brownish substance called tar meanwhile electric cigarette contains of many chemical substances.
\end{abstract}

Results: Data were recorded after exposure of cirarettes's and analyzed by statistic test used Independent sampe $T$ test whit the significance level $a=0.05$. Cigarette smoke affects the color of acrylic artificial teeth. Meanwhile, electric cigarette effect the color of acrylic teeth but insignificantly. There are artifisial teeth color discoloration different between exposure of cigarette's and electric cigarette's smoke with $p=0.010<a=0.05$.

Conclusion: This study shows that there is a discoloration difference between acrylic artificial teeth exposed to filter- and electroniccigarette smoke
Department of Prosthodontic, Faculty of Dentistry, Muslim University of Indonesia, Makassar, Indonesia
*Correspondence to: Chusnul Chotimah, Department of Prosthodontic, Faculty of Dentistry, Muslim University of Indonesia, Makassar, Indonesia chusnulchotimah70@gmail.com

Received: 25 September 2018 Revised: 10 November 2018 Accepted: 9 August 2020 Available Online: 1 December 2020

Keywords: Artificial, Cigarette, Discoloration

Cite this Article: Chotimah C, Ramadan MA. 2020. Discoloration difference between acrylic artificial teeth exposed to filter- and electroniccigarette smoke in the Laboratory Faculty of Dentistry, Muslim University of Indonesia in 2017. Journal of Dentomaxillofacial Science 5(3): 177-180. DOl: $10.15562 / j \mathrm{dmfs} . v 5 \mathrm{i} 3.821$

\section{Introduction}

Tooth loss is a change in the oral cavity which may be caused by low dental and oral health status, such as caries and periodontal disease. Moreover, tooth loss may also be caused by trauma, systemic problems, sociodemographic conditions and individual habits. ${ }^{1}$ If not immediately replaced, the missing teeth may cause difficulties to the patients themselves, such as in chewing food, as well as dental supraeruption, tilting and shifting. ${ }^{2}$ Therefore a denture used to restore the disturbed functions. ${ }^{3}$ According to the RISKESDAS survey in 2007, the prevalence of denture users in Indonesia was only approximately $4.5 \% .{ }^{4}$ Denture users in South Sulawesi was reached $4.8 \%$, whereas particularly in the city of Makassar the prevalence was reached $2.9 \%$.

Dentures can be divided into fixed- and removable-denture. All denture types have artificial tooth components. Artificial teeth can be fabricated from various type of material, namely porcelain, and acrylic resin or polymethyl methacrylate (PMMA) which most often used as basic material of artificial teeth. Acrylic has different properties from porcelain, especially in the case of retaining stain. Acrylic more prone to be discolored by stain compared to porcelain. Stain attached to the acrylic artificial teeth may be derived from foods or drinks, and may be acquired from the smoking habits. ${ }^{5}$

Smoking is a habit which we commonly encounter anywhere in the world. This habit has been broadly carried in both high- and low-educated environment. According to the World Health Organization (WHO), cigarette smoke environment is the cause of various diseases and can also affect healthy non-smoker individuals. ${ }^{6}$

The prevalence of smoking in the world is still high. In the UK, adult smoker population was estimated to be 12.5 million, carried by $27 \%$ men and $24 \%$ women. In the US, smoking was reported carried by $26.4 \%$ men and $22 \%$ women. In Indonesia, smoking prevalence in men appears even higher with $50-70 \%$ percentage, while in women $3 \% .{ }^{6}$ In the Province of South Sulawesi, population aged 10 years and over who smoke everyday reached $20.9 \%$. And according to the survey, the types of cigarettes are often consumed include clove- and filter-cigarettes.

Kretek- and filter- or white-cigarettes are cigarettes made from tobacco as their basic material. Smoke from tobacco cigarettes contains 
various dangerous materials which may have negative impacts on health. As a result of the multitude negative impacts of cigarettes and filter cigarettes, cigarettes then made electronically. ${ }^{5}$

Electronic cigarette or e-cigarette is a nicotine replacement therapy which uses electricity from battery power to deliver nicotine in the form of steam and called by WHO as electronic nicotine delivery system. Electronic-cigarette designed to provide nicotine without the need of tobacco burning and to give a smoking sensation to the users.

The chemical compound content and the high temperature of cigarette smoke can cause local interferences in the oral cavity. Brownish black stain is derived from tobacco sap produced during tobacco burning. It causes aesthetic problems. ${ }^{6}$

\section{Material and Methods}

The type of research used here is true experimental. This research said be a true experimental research because its randomization was carried out on the sample. Post test results used to see the discoloration difference between acrylic artificial teeth exposed to filter- and electronic-cigarette smoke. The research was conducted in the Laboratory of Dentistry Faculty at the Muslim University of Indonesia for a day. It was conducted on December 2017.

The sample used here is acrylic artificial upper anterior teeth based on the reason that the teeth are most anteriorly seated teeth on which the aesthetic value is very important. Number of samples to be used in this study was determined by using total sampling, where only objects meet the inclusion criteria were sampled. In this case, only upper anterior teeth were used. Based on this, a sample group consisted of six artificial teeth. Sample groups consist of group 1 which were exposed to filter-cigarette smoke and group 2 which were exposed to electronic-cigarette smoke.

This research was conducted by the direct exposure to electronic- and filter-cigarette smoke on different artificial teeth using a smoking simulator. Assessment of color samples was measured using a standardized international color measurement system adopted by Commission Internationale d'Eclairge (CIE) namely CIE $L^{\star} a^{\star} b$ system.

Data obtained were then processed using SPSS program and statistically tested using independent sample T-test after considering the involvement of the two artificial acrylic teeth sample groups.

\section{Results}

Discoloration difference between acrylic artificial teeth exposed to filter- and electronic-cigarette smoke was completely evaluated. The research was conducted in the Laboratory of Dentistry Faculty at the Muslim University of Indonesia for a day on December 1, 2017. The data were then analyzed using SPSS program and tested using independent sample T-test with significance level $a=0.05$. Results are shown in the distribution table 1 , table 2 and table 3.

On the statistical test using independent sample T-test, the value of $\mathrm{p}=0.010<\alpha=0.05$ were obtained, which means that there was a discoloration difference between acrylic artificial teeth exposed to filter- and electronic- cigarette smoke.

\section{Discussion}

Results of this study regarding the discoloration difference between acrylic artificial teeth exposed to filter- and electronic-cigarette smoke shows that the samples exposed to filter-cigarette smoke obtained a lower mean post- exposure (L) value 62.83 than its

\section{Table 1 Represents discoloration of the artificial teeth exposed to filter-cigarette smoke from all six samples; the mean pre- and post-exposure (L) values are 73 and 62.83, respectively, the mean pre- and post-exposure (a) values are -1 and 2, respectively, and the mean pre- and post-exposure (b) values are 12 and 24, respectively, therefore the mean value of $\Delta E^{*} a b=16.47$ finally obtained}

\begin{tabular}{|c|c|c|c|c|c|c|c|c|}
\hline & \multirow[b]{2}{*}{ Sampel } & \multicolumn{3}{|c|}{ Pre-exposure } & \multicolumn{3}{|c|}{ Post-exposure } & \multirow[b]{2}{*}{$\left(\Delta E^{*} \mathrm{ab}\right)$} \\
\hline & & $\mathbf{L}$ & a & b & $\mathbf{L}$ & a & b & \\
\hline \multirow{6}{*}{ Filter cigarette } & 1 & 73 & -1 & 12 & 69 & 0 & 22 & 10.82 \\
\hline & 2 & 73 & -1 & 12 & 70 & 0 & 21 & 9.54 \\
\hline & 3 & 73 & -1 & 12 & 58 & 7 & 34 & 27.80 \\
\hline & 4 & 73 & -1 & 12 & 61 & 1 & 24 & 17.09 \\
\hline & 5 & 73 & -1 & 12 & 58 & 2 & 22 & 18.28 \\
\hline & 6 & 73 & -1 & 12 & 61 & 2 & 21 & 15.30 \\
\hline Mean & & 73 & -1 & 12 & 62.83 & 2.00 & 24.00 & 16.47 \\
\hline
\end{tabular}


Table 2 Represents discoloration of the artificial teeth exposed to electronic-cigarette smoke from all six samples; the mean pre- and post-exposure (L) values are 73 and 70.83, respectively, the mean pre- and post-exposure (a) values are -1 and -1 , respectively, and the mean pre- and post-exposure (b) values are 12 and 19.5, respectively, therefore the mean value of $\Delta E^{*} a b=6.81$ finally obtained

\begin{tabular}{|c|c|c|c|c|c|c|c|c|}
\hline & \multirow[b]{2}{*}{ Sampel } & \multicolumn{3}{|c|}{ Pre-exposure } & \multicolumn{3}{|c|}{ Post-exposure } & \multirow[b]{2}{*}{$\left(\Delta E^{*} \mathrm{ab}\right)$} \\
\hline & & $\mathbf{L}$ & $\mathbf{a}$ & b & $\mathbf{L}$ & $\mathbf{a}$ & b & \\
\hline \multirow{6}{*}{ Filter cigarette } & 1 & 73 & -1 & 12 & 76 & -2 & 17 & 5.92 \\
\hline & 2 & 73 & -1 & 12 & 69 & -1 & 20 & 4.90 \\
\hline & 3 & 73 & -1 & 12 & 73 & -1 & 20 & 2.83 \\
\hline & 4 & 73 & -1 & 12 & 68 & 0 & 20 & 9.49 \\
\hline & 5 & 73 & -1 & 12 & 67 & 0 & 23 & 12.57 \\
\hline & 6 & 73 & -1 & 12 & 72 & -2 & 17 & 5.20 \\
\hline Mean & & 73 & -1 & 12 & 70.83 & -1 & 19.5 & 6.81 \\
\hline
\end{tabular}

Table 3 Describes the discoloration difference between acrylic artificial teeth exposed to filter- and electronic-cigarette smoke; from the two sample groups studied, means and SDs obtained were $16.47 \pm 6.53$ and $6.81 \pm 3.55$ for filter- and electroniccigarette groups, respectively

\begin{tabular}{ccccc}
\hline & Group & $\mathbf{n}$ & Mean \pm SD & Nilai P \\
\hline \multirow{2}{*}{$\Delta \mathrm{E}^{*} \mathrm{ab}$} & Filter cigarette & 6 & $16.47 \pm 6.53$ & 0.010 \\
& Electric cigarette & 6 & $6.81 \pm 3.55$ & \\
\hline
\end{tabular}

Independent sample $\mathrm{T}$ test: $\mathrm{p}<0,05$ : significant

pre-exposure value 73 , a negative $\Delta \mathrm{L}$ value which indicates that the sample became darker. The mean post-exposure (a) value (2) was higher than its pre-exposure value $(-1)$, a positive $\Delta$ a value which indicates that the sample became more reddish in color than before. Then the mean post-exposure (b) value (24) was higher than its pre-exposure value (12), a positive $\Delta b$ value which indicates that the sample became more yellowish in color than before. The samples exposed to electronic-cigarette smoke obtained a lower mean post-exposure (L) value 70.83 than its pre-exposure value 73 , a negative $\Delta \mathrm{L}$ value which indicates that the sample became darker. The mean post-exposure (a) value -1 was as high as its pre-exposure value -1 , which means that there was no change in $\Delta \mathrm{a}$ value. Then the mean post-exposure $b$ value 19.5 was higher than its pre-exposure value 12 , a positive $\Delta \mathrm{b}$ value which indicates that the sample became more yellowish in color than before. From the results above, the mean $\Delta \mathrm{E}^{\star}$ ab value obtained was 16.47 for filter-cigarette and 6.81 for electric-cigarette, which means that there was a discoloration difference between acrylic artificial teeth exposed to filter- and electroniccigarette smoke. Based on the statistical tests using independent sample T-test, the value of $p=0.010$ $<\alpha=0.05$ were obtained, thus it was decided that Ho rejected and $\mathrm{Ha}$ accepted, which means that there is a discoloration difference between acrylic artificial teeth exposed to filter- and electronic-cigarette smoke in the Laboratory of Dentistry Faculty at the Muslim University of Indonesia in 2017.

It was found in this study that the color of the artificial teeth exposed to filter-cigarette smoke looks darker compared to the color of ones exposed to electronic-cigarette smoke. This is because of the tobacco burning in the filter-cigarette which immediately produce smoke containing tobacco sap that may leave a brownish black colored stain. Moreover, filter-cigarette also contains nicotine which capable of causing color changes, and tar, a brown sticky substance which may leave a brown or yellow stain on artificial teeth; while the electronic-cigarette has no tar content and operates by warming tobacco using electricity from battery power, instead of burning it, to deliver nicotine in the form of steam, thus causing a color change which not as significant as discoloration produced by filter-cigarette smoke exposure.

The results of this study are in line with the findings of, ${ }^{5}$ where the artificial tooth discoloration did occur after cigarette smoke exposure because of its tar content and heat generated. Artificial tooth discoloration occurred in this study also in line with the results of a study by Kohli et al. ${ }^{8}$ which found that color change may occur due to the sample environmental conditions and the ability of exposing material to change color or leave its 
stain. The results found a distinctive discoloration when acrylic artificial teeth exposed to electroniccigarette smoke; the color change was caused by the absorption of water from the electric-cigarette steam by the artificial teeth; this is in line with the results of a study by Amin. ${ }^{9}$ which explained that PMMA is a hydrophilic material which may easily binds to water and consequently lack its color stability. Artificial teeth used in this study were discolored due to cigarette smoke exposure, and explained that one of the disadvantages of acrylic resin laid on its pores which can absorb water, liquid, food scraps or chemical substances; and the presence of the polymer chain content would become unfavorable to the material itself because it would form a network which may absorb or bind foreign objects. The color change occurred as a result of cigarette smoke exposure explained that the tar delivered by cigarette smoke contains aromatic hydrocarbon. This surface solvent substance is a contributing factor fordiscoloration. In this study,there is a discoloration difference between acrylic artificial teeth exposed to filter- and electronic-cigarette smoke; that lower discoloration value resulted by electronic-cigarette smoke exposure whereas higher discoloration value resulted by filter-cigarette smoke exposure. Thisdiscoloration rate difference is a result of the difference in discolorizing substancecontent between electric- and filter-cigarette. Electroniccigarette smoke causes less color change because of its less discolorizing substance content compared to filter-cigarette smoke. ${ }^{10,11}$

\section{Conclusion}

This study shows that there is a discoloration difference between acrylic artificial teeth exposed to filter- and electronic-cigarette smoke, with the value of $p=(0.010)<\alpha=0.05$ which means that the color of the artificial teeth exposed to filtercigarette smoke looks darker compared to the color of ones exposed to electronic-cigarette smoke as a result of less discolorizing substance contained in the electronic- compared to filter- cigarette smoke.

\section{Acknowledgment}

Thank you for Faculty of Dentistry, Muslim University of Indonesia for the direction and support of this research.

\section{Conflict of Interest}

The authors report no conflict of interest.

\section{References}

1. Khalifah N. Factor associated with loss and prosthodontic status among sudanese adults. J Oral Sci 2012;54: 303.

2. Prabowo DMS, Widodo HB. Nicotine stomatitis in smokers: a case report. J Dentomaxillofac Sci 2018;3: 58-60.

3. Bortoluzzi MC, Traebert J, Lasta R, et al. Tooth loss, chewing ability and quality of life. Contemp Clin Dent 2012;3: 393-397.

4. Garcia. Effect of staining sulutions and repolishing on color stability of direct compistes. J Appl Oral Sci Brazil 20101;8: 249- 254.

5. Patil SS. Effect of cigarette smoke on acrylic resin teeth. J Clin Diag Res 2013;7: 2056-2059.

6. Khalisa E. The relationship of smoking habits with the formation of stains (tooth stains) in patients at the dental clinic Ratu Zalecha Martapura Hospital. Dentino J Kedokteran Gigi 2016;1: 28. (In Indonesia)

7. Tanuwihardja RK, Susanto AD., Electric cigarette (electronic cigarette). J Respir Indo 2012;323: 53-61. (In Indonesia)

8. Kohli S, Bhatia S. Evaluation of the color durability of acrylic resin veneer materials after immersion in common beverages at different time intervals: a spectrophotometric study. Biomed J 2015;38: 7-8.

9. Amin F. Spectrophotometric assessment of color changes of heat cure acrylic resins after exposure to commonly consumed beverages. J Dow Univ Health Sci Karachi 2014;8: 62-64.

10. Yam KL, Papadakis SE. A simple Digital Imaging Method for Measuring and annalyzing color of food surface. J Food Eng 2004;61: 137-142.

11. Prabowo DMS, Widodo HB. Nicotine stomatitis in smokers: a case report. J Dentomaxillofac Sci 2018;3: 58-60.

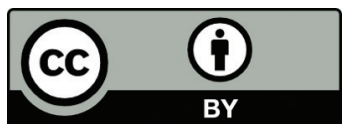

This work is licensed under a Creative Commons Attribution 